\title{
Alterações cardiorrespiratórias de idosos portadores da síndrome da imunodeficiência humana adquirida
}

\author{
Cardiorespiratory changes and QoL \\ of elderly persons with AIDS
}

FisiSenectus. Unochapecó Ano 1, n. 1 - Jan./Jun. 2013 p. 25-34

\section{Lilian Marin \\ Mestranda em Envelhecimento Humano pela Universidade de Passo Fundo, docente da Universidade Comunitária da Região de Chapecó.}

\section{Camila Aparecida de Campos Pinto}

Fisioterapeuta, graduada pela Universidade Comunitária da Região de Chapecó.

\section{Kéuri Dalmoro}

Fisioterapeuta, graduada pela Universidade Comunitária da Região de Chapecó.

\begin{abstract}
Resumo
Objetivo: avaliar as condições do sistema cardiorrespiratório de idosos portadores da síndrome da imunodeficiência humana. Métodos: neste estudo quantitativo transversal foram avaliados oito idosos portadores da AIDS com $65 \pm 5,88$ anos, PAS $121,25 \pm 16,42$ $\mathrm{mmHg}, F C 72,50 \pm 12,25 \mathrm{bpm}$, IMC de 23,12 $\pm 3,61 \mathrm{~kg} / \mathrm{m} 2$ e analisados valores da Escala Analógica Visual (EAV) e da Escala do Medical Research Council (MRC); do Pico de Fluxo Expiratório (PEF) e das Pressões Inspiratória e Expiratória Máximas (PImáx e PEmáx). Foi utilizado o coeficiente de correlação de Pearson para algumas variáveis, com valor de $p<0,05$. Resultados: o comportamento da PAS e $F C(r=0,89)$ demonstrou uma correlação forte positiva; a média da expansibilidade torácica dos voluntários apresentou valores de $3,06 \mathrm{~cm}$ na região axilar, $2,63 \mathrm{~cm}$ na região xifoide e $1,31 \mathrm{~cm}$ na região abdominal; as médias de PFE, PEmáx e PImáx estavam abaixo dos esperado para idade e sexo, respectivamente $305 \pm 153,34,65 \pm 37,80$ e 66,25 $\pm 43,07$ (PFE x PEmáx: $r=0,35$ ); a média da dispneia obteve sintomas leves referidos, $3 \pm 2,56$ no repouso e 1,63 $\pm 1,30$ ao esforço. Conclusão: foram encontrados dados estatisticamente válidos do comportamento da PAS e FC e do PFE e PEmáx, que provavelmente podem estar relacionados ao processo de envelhecimento dos indivíduos e à administração de medicamentos para terapia antirretroviral.
\end{abstract}

\section{Palavras-chave}

AIDS. Fisioterapia. Geriatria. 


\begin{abstract}
Objective: to evaluate the conditions of the cardiorespiratory system and quality of life of elderly patients with the acquired immunodeficiency syndrome. Methods: this cross-sectional quantitative study evaluated eight elderly patients with AIDS $65 \pm 5.88$ years, SBP $121,25 \pm 16.42 \mathrm{mmHg}, \mathrm{HR} 72,50 \pm 12.25 \mathrm{bpm}, \mathrm{BMI} 23.12 \pm 3.61$ $\mathrm{kg} / \mathrm{m} 2$ and analyzed values of the Visual Analogue Scale (VAS) and Scale of the Medical Research Council (MRC); of Peak Expiratory Flow (PEF) and maximal inspiratory and expiratory pressures (MIP and MEP). The Pearson correlation coefficient was used for some variables, with $p<0.05$. Results: the SBP and HR $(r=0.89)$ showed a strong positive correlation, the average chest expansion of the volunteers had values of $3,06 \mathrm{~cm}$ at the axillary region, the region $2,63 \mathrm{~cm}$ and $1,31 \mathrm{~cm}$ at the xiphoid region abdominal; the mean PEF, MEP and MIP were below the expected for age and gender, respectively $305 \pm 153,34,65 \pm 37,80$ and $66,25 \pm 43.07$ (PEF X MEP: $r=0.35$ ); mean dyspnea got mild symptoms above, $3 \pm 2,56$ at home and $1,63 \pm 1,30$ to the effort. Conclusion: we found no statistically valid data of SBP and HR and MEP and PEF, which probably can be related to the aging process of individuals and the administration of drugs for antiretroviral therapy.
\end{abstract}

\title{
Keywords
}

AIDS. Physical therapy. Geriatric.

\section{Introdução}

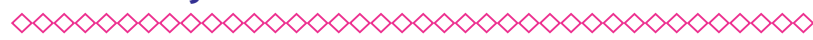

As tendências demográficas indicam o aumento do número de indivíduos idosos em relação aos indivíduos jovens, não só nos países desenvolvidos, mas também nos países em desenvolvimento, podendo, desta forma, incluir o Brasil ${ }^{1,2}$. Esse acréscimo da população idosa se deve em especial a redução da mortalidade e fecundidade, logo, as alterações demográficas são responsáveis pelo envelhecimento populacional ${ }^{3}$.

0 termo velhice constitui-se em um processo dinâmico e progressivo, em que há alterações tanto morfológicas, fisiológicas, bioquímicas, psicológicas, quanto no âmbito social, que determinam a perda gradual da capacidade e funcionalidade do indivíduo em se adaptar ao meio ambiente, acarretando maior incidência de processos patológicos 4 . Já no aspecto biológico do envelhecimento humano, há expressivas modificações em vários órgãos e sistemas corporais, dentre eles podemos destacar o sistema imunológico ${ }^{3}$.

À medida que os indivíduos envelhecem, a capacidade do sistema imunológico (linfócito T e anticorpos) diminui tanto de forma qualitativa quanto quantitativa ${ }^{2,3}$. Assim, as respostas imunológicas tornam-se reduzidas e atuam de forma inadequada em reconhecer os próprios elementos do organismo e ainda os estranhos, resultando em aumentos significativos de doenças autoimunes e infectocontagiosas ${ }^{2,3,5}$. Dados estatísticos revelam que há o crescimento do número de infeç̧ões por HIV/AIDS em pessoas com 60 anos ou mais, o que representa $2,1 \%$ da totalidade dos casos registrados no País ${ }^{6}$. Em uma pesquisa realizada pela Fundação Perseu Abramo, em parceria com o SESC nacional e de São Paulo, em 2006, com 2.136 idosos distribuídos em 204 municípios (pequenos, médios e grandes), dispostos nas cinco macrorregiões do Brasil, quanto a exames rotineiros o teste de HIV nunca foi realizado pela grande maioria dos idosos $(86 \%)^{7}$.

A forma de infecção pelo HIV no indivíduo idoso apresenta particularidades inerentes ao enveIhecimento do organismo, observando-se um curso clínico mais rápido, com manifestações mais precoces e severas, número mais elevado de infecções e/ou neoplasias oportunistas, provavelmente relacionadas com as comorbidades e a imunosenescência ${ }^{8}$.

Percebe-se, assim, que as doenças que acometem os sistema pulmonar e o cardiovascular continuam sendo as causas mais comuns de doença e morte em pacientes com infeç̧ão por HIV ${ }^{9}$, principalmente as infeç̧ões oportunistas e bacterianas ${ }^{10}$, uma vez que o envelhecimento leva a inúmeras alterações pulmonares e cardíacas, que ocasionam o enrijecimento da caixa torácica, fraqueza da musculatura respiratória, diminuição da 
complacência nos pulmões, hipertrofia miocárdica, alteração da complacência ventricular, entre outras ${ }^{4}$.

Considerando que o indivíduo com AIDS possui uma imunodepressão progressiva e que as afecções cardíacas e respiratórias são as que mais acometem essa população, o presente estudo objetivou avaliar as condições do sistema cardiorrespiratório de idosos portadores da síndrome da imunodeficiência humana.

\section{Materiais e métodos}

$\infty \times \infty \times \infty \times \infty \times \infty \times \infty \times \infty \times \infty \times \infty \times \infty \times \infty \times \infty)$

Trata-se de um estudo quantitativo de abordagem transversal conduzido em um centro especializado no atendimento a pacientes com HIV/AIDS, pertencente à rede pública, vinculado ao Sistema Único de Saúde da cidade de Chapecó (SC), com base na avaliação cardiorrespiratória de idosos com AIDS, no período de abril a junho de 2010.

Para seleção dos pacientes, adotou-se como critérios de inclusão ser idoso com idade igual ou superior a 60 anos (conforme o Estatuto do Idoso, Lei n. 10.741 , de $1^{\circ}$ de outubro de 2003), apresentar sorologia positiva para o HIV, estar administrando terapia antirretroviral, frequentar periodicamente o centro especializado, residir na cidade de Chapecó (SC) e aceitar participar da pesquisa. Como critérios de exclusão: presença de problemas mentais ou fatores que interferissem na avaliação.

A população do estudo foi levantada por intermédio do banco de informações do próprio centro, representando um total de 24 idosos, dos quais 19 atendiam aos critérios de inclusão. Porém, dez idosos recusaram-se a participar do estudo e um encontrava-se hospitalizado durante o período de coleta de dados, ficando a amostra composta por oito idosos.

O estudo foi aprovado pelo Comitê de Ética em Pesquisa da Universidade Comunitária da Região de Chapecó (Unochapecó) sob o protocolo n. 276/09. Todos os participantes foram devidamente esclarecidos sobre a pesquisa e assinaram o Termo de Consentimento Livre e Esclarecido, seguindo as exigências para pesquisas que envol- vem seres humanos, de acordo com a Resolução $\mathrm{n}$. 196/96 do Conselho Nacional de Saúde.

Para a coleta de dados foram utilizados: uma ficha de avaliação em fisioterapia cardiorrespiratória elaborada de acordo com a literatura ${ }^{11,12}$; utilizou-se também a Escala Analógica Visual (EAV) e a Escala do Medical Research Council (MRC) ambas para avaliar o grau de dispneia.

A Ficha de Avaliação em Fisioterapia cardiorrespiratória abrangeu questões referentes à anamnese e à avaliação do indivíduo quanto aos aspectos físicos e subjetivos. Foram coletadas informações pessoais como: idade, gênero, cor; história da doença pregressa e atual, presença de sintomas ou doenças associadas, cirurgias cardíacas ou pulmonares prévias, tabagismo, etilismo, sedentarismo, uso de medicamentos, pressão arterial sistólica e diastólica (PAS e PAD), frequência cardíaca ( $F C$ ) em batimentos por minuto (bpm), frequência respiratória (fr) em respirações por minuto (rpm), saturação de oxigênio $\left(\mathrm{SatO}_{2}\right)$, ausculta pulmonar (AP), massa corporal $(\mathrm{Kg})$, estatura $(\mathrm{m})$, índice de massa corporal (IMC), circunferência da cintura e do quadril, relação da cintura e quadril (RCQ), tipo do padrão respiratório e cirtometria tóraco-abdominal, pico de fluxo expiratório (PEF) e pressões inspiratória e expiratória Máximas (PImáx e PEmáx). Para isso, foram utilizados: esfigmomanômetro e estetoscópio Premium ${ }^{\circledR}$, oxímetro de dedo Nonin, modelo Ônix $950 \circledR$, fita métrica convencional em centímetros, marca ISP.

Especificadamente na observação do padrão respiratório, foram considerados o diafragmático, o intercostal superior e o paradoxal; determinado, respectivamente, quando o sujeito possui predomínio da elevação do abdômen em relação ao tórax ou vice-versa, ou quando há assincronia entre o tórax e o abdômen ${ }^{13}$. A cirtometria tóraco-abdominal foi realizada medindo-se os níveis axilar, xifoideano e abdominal, com o indivíduo sentado sobre uma maca, sendo o diâmetro da caixa torácica mensurado no repouso respiratório, ao final da inspiração máxima (Imáx) e ao final da expiração máxima (Emáx) ${ }^{14}$.

A EAV consistia em uma linha horizontal demarcada em suas extremidades os valores 0 e 10 para obter o grau de dispneia referida pelo indi- 
víduo em repouso. Estudos demonstram que esta escala possui um grande potencial em fornecer um sistema de medida confiável e sensível a dispneia ${ }^{15}$. Já a MRC trata-se de uma escala a qual classifica a dispneia em cinco categorias, sendo que o indivíduo escolhe o item correspondente a quanto a dispneia limita suas atividades de vida diária ${ }^{16}$.

Para avaliação da PFE, utilizou-se o ASSESS $\circledast$ Peak Flow Meter (60-880L/min), da marca Respironics ${ }^{\circledR}$, que consiste em um pequeno aparelho portátil feito de material plástico transparente, contendo uma graduação de valores que avalia a saída do pico de fluxo de ar de dentro dos pulmões em litros por minuto (L/min). 0 indivíduo permaneceu em posição ortostática, com coluna cervical alinhada e a boca acoplada em um bucal descartável de maneira a evitar desvio do ar, e realizou uma expiração máxima, repetindo a manobra por mais duas vezes, dando um intervalo de um minuto entre uma expiração e outra para descanso ${ }^{17}$. Considerou-se o valor da melhor tentativa entre as três.

A mensuração da PImáx e PEmáx foi realizada utilizando o Manovacuômetro Comercial Médica ${ }^{\circledR}$, que possui intervalos de $4 \mathrm{cmH} 20$ e variações de $+/-120 \mathrm{cmH2O}$, em que o indivíduo permaneceu sentando em uma cadeira, com coluna cervical alinhada, clipe nasal, orientou-se acoplar a boca no bucal para evitar desvio do ar e realizou três inspirações máximas e após três expirações máximas (com intervalo de um minuto entre cada inspiração e um minuto entre cada expiração para descanso, sendo considerado o maior valor) ${ }^{18}$. Para avaliação dos valores esperados da manuovacuometria, utilizou-se os critérios de Neder et al. ${ }^{19}$ : Homens PImáx $=-0,8 x$ idade $+155,3 /$ PEmáx $=-0,8 x$ idade + 165,3; Mulheres - PImáx $=-0,49 x$ idade $+110,4$ / PEmáx: $-0,61 \mathrm{x}$ idade $+155,6$.

Os dados coletados foram tabulados e decodificados em planilha do Microsoft Office Excel® versão 2003 e do BioEstat $5.0{ }^{\circledR}$. Para descrever os valores encontrados na pesquisa, utilizou-se porcentagem, média e desvio-padrão, apresentados na forma de gráfico e tabelas. E ainda para a determinação das relações entre algumas variáveis, foram utilizou-se os coeficientes de correlação de Pearson, com valor de $p<0,05$ (que determina o grau de associação entre as variáveis cruzadas).

\section{Resultados}

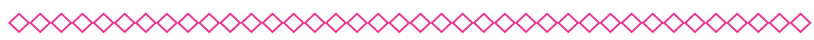

$\mathrm{Na}$ análise dos dados, dos oito idosos avaliados, a idade média foi de $65 \pm 5,88$ anos, sendo quatro do gênero feminino (50\%) e quatro do masculino $(50 \%)$, cinco pardos $(62,5 \%)$ e três brancos (37,5\%), dois tabagistas (25\%), nenhum etilista e seis sedentários (75\%), PAS 121,25 $\pm 16,42 \mathrm{mmHg}$, PAD 78,75 $\pm 9,91 \mathrm{mmHg}$, FC 72,50 $\pm 12,25 \mathrm{bpm}$, fr de $15 \pm 2,83 \mathrm{rpm}$, SatO 2 de $96 \pm 0,03 \%$, massa corporal de $64,06 \pm 18,35 \mathrm{Kg}$, altura de $1,65 \pm 0,12 \mathrm{~m}$, IMC de $23,12 \pm 3,61 \mathrm{Kg} / \mathrm{m}^{2}$, circunferência da cintura e quadril das mulheres, respectivamente, $78,5 \pm 9,95 \mathrm{~cm}$ e $93,25 \pm 5,12 \mathrm{~cm}, \mathrm{RCQ}$ das mulheres de $0,83 \pm 0,08$, circunferência da cintura e quadril dos homens, respectivamente, $101,75 \pm 10,81$ e $102,25 \pm 9,00$, RCQ dos homens de 0,99 $\pm 0,05$.

O comportamento da PAS e FC dos idosos avaliados em repouso é mostrado na tabela 1 , atingindo um coeficiente de Pearson $r=0,89(p=0,0029)$, demonstrando uma correlação forte positiva entre as duas variáveis.

$\mathrm{Na}$ ausculta pulmonar, todos os idosos apresentaram alteração no murmúrio vesicular (MV), sendo que três $(37,5 \%)$ indivíduos apresentaram diminuição do MV em todos os campos pulmonares; três $(37,5 \%)$ apresentaram diminuição do MV em bases pulmonares; dois (25\%) apresentaram MV abolido em bases pulmonares; um (12,5\%) apresentou MV abolido em médio do pulmão direito; dois (25\%) apresentaram diminuição do MV em médio de ambos os pulmões; e um $(12,5 \%)$ apresentou diminuição do MV em ápice do pulmão. Apenas um idoso (12,5\%) apresentou ruídos adventícios na AP (presença de estertores crepitantes em médio do pulmão esquerdo).

Quanto ao padrão respiratório, cinco idosos $(62,5 \%)$ apresentaram padrão intercostal superior; três idosos $(37,5 \%)$ apresentaram padrão abdominal; e o padrão paradoxal não foi observado em nenhum voluntário.

Em relação à cirtometria tóraco-abdominal, conforme mostra a tabela 1 , pode-se constatar que a média dos valores obtidos nas regiões axilar, xifoideana e abdominal apresentaram mínima diferença na avaliação em repouso, Imáx e Emáx, evidenciando a diminuição da mobilidade tóraco- 
-abdominal nos idosos estudados. A média da expansibilidade torácica dos voluntários apresentou valores de $3,06 \mathrm{~cm}$ na região axilar, 2,63 cm na região xifoide e $1,31 \mathrm{~cm}$ na região abdominal. Analisando os valores individuais da tabela 1 , observa-se que o idoso 5 não obteve uma expansão tóraco-abdominal maior que $1 \mathrm{~cm}$ em todos os níveis avaliados; por outro lado, o idoso 3 atingiu na Imáx da região axilar e xifoideana diferença de $4 \mathrm{~cm}$ em relação ao repouso, e na Emáx $2 \mathrm{~cm}$ nas mesmas regiões.

Conforme mostra a tabela 2, pode-se perceber que apenas o idoso 1 atingiu o PFE estimando para sua idade e altura, observado ainda que os idosos 5 e 8 atingiram um valor de aproximadamente $70 \%$ baixo do estimado, dados estes comparados com a tabela que acompanhava o manual aparelho. Para a PEmáx foi possivel identificar que somente dois idosos atingiram o valor estimado, sendo eles o 1 e 7, e os demais mantiveram os valores a abaixo do estimado, principalmente o idoso 2. Para a PImáx foi possível identificar que três idosos atingiram 0 valor estimado, sendo eles o 3, 4 e 7, e os demais mantiveram-se abaixo do estimado, ressaltando-se o idoso 5, com valor muito abaixo do estimado.

Na avaliação da dispneia, segundo a EAV, pode-se constatar que apenas o idoso 4 não apresentou nenhum grau de dispneia. 0 idoso 3 referiu dispneia grau 1 e os idosos 1, 2 e 6 referiram dispneia grau 2, ambos considerados leves, segundo dados da escala; o idoso 5 referiu dispneia grau 8, sendo considerado um valor intenso. Na MRV novamente um não referiu dispneia, o idoso 04, porém quatro idosos referiram dispneia grau 1, considerada somente durante a realização de exercício intenso, sendo eles o idoso 1, 2, 3 e 5 . 0 idoso 5 referiu dispneia grau 4 ou aquela que o idoso necessita parar para respirar depois de ter andado menos de 100 metros ou após alguns minutos (tabela 3).

\section{Discussão}

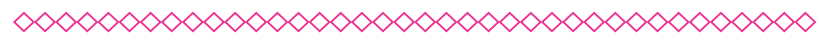

De acordo com a literatura, idosos infectados pelo HIV frequentemente são acometidos por doenças que envolvam o sistema cardiorrespiratório, sobretudo quando há associação de outras comorbidades comuns do envelhecimento ${ }^{20}$. Nes- te contexto, a avaliação das condições do sistema cardiorrespiratório realizada nos voluntários da pesquisa pode auxiliar na melhor compreensão deste tema, pois permite a análise de variáveis pouco difundidas na literatura.

O número de casos de AIDS notificados no Brasil em indivíduos com 60 anos ou mais, de 1980 a junho de 2009, é de 10.886 pessoas, o que corresponde a $2,35 \%$ do total geral, sendo que destes $67,1 \%$ são do sexo masculino e $32,9 \%$ do sexo feminino. Verifica-se que há predomínio de homens infectados e de indivíduos da cor branca, diferente dos resultados obtidos nesta pesquisa, considerando o número restrito da amostra ${ }^{21}$.

Grande parte da amostra estudada caracterizou-se pelo sedentarismo. Sabe-se que mudanças no estilo de vida são estudadas amplamente na população geral com o objetivo de analisar as condições cardiorrespiratórias. Em um estudo de revisão que abordou a prática de atividade física em indivíduos portadores do vírus HIV, estes relataram que há melhoria na condição cardiorrespiratória e aumentos sugestivos da contagem de linfócitos $\mathrm{CD} 4+$, quando o indivíduo realiza regularmente atividade física ${ }^{22}$.

A média da PA, FC, FR e S02, assim como do IMC e RCQ para ambos os gêneros, mostrou-se dentro dos parâmetros de normalidade ${ }^{23}$. Apesar dos idosos estudados não apresentarem alterações das variáveis citadas, a maioria possui um estilo de vida sedentário, o que pode causar prejuízos à saúde, uma vez que a imunodepressão e valores limítrofes de LCD4+ propiciam a manifestação de doenças oportunistas ${ }^{10}$, sendo as doenças pulmonares uma das causas mais comuns de distúrbios agudos e óbito em pacientes infectados pelo HIV ${ }^{24}$.

De acordo com a tabela 1, quanto maior a PAS, maior a FC dos idosos infectados pelo HIV. A literatura relata que há forte correlação entre 0 aumento da PAS x FC com o risco para desenvolver cardiomiopatias ${ }^{25}$. A amostra estudada não apresentou valores médios de PAS e FC acima dos parâmetros de normalidade. Contudo, deve-se considerar o efeito provocado pela terapia antirretroviral (TARV), pois estudos demonstraram que há aumento do risco cardiovascular devido às complicações metabólicas potencialmente atero- 
gênicas associadas a esta terapia ${ }^{26}$. Em uma pesquisa ${ }^{27}$, que avaliaram o risco cardiovascular pré e pós-TARV em paciente com AIDS, observou-se que o grupo com maior risco significativo de doenças cardiovasculares é aquele com idade entre 60 e 75 anos. Observou-se que os indivíduos que apresentaram os maiores valores de PAS e FC $(n=2)$ eram homens, sedentários e estavam entre aqueles $(n=3)$ com maior índice de dispneia referida no repouso e ao esforço.

A cirtometria revelou que a média da expansibilidade torácica dos voluntários encontra-se abaixo da normalidade descrita por Pryor e Weber ${ }^{11}$ de $\geq 5 \mathrm{~cm}$, podendo decair lentamente aproximadamente $2,5 \mathrm{~cm}$ em indivíduos maiores de 74 anos. No estudo realizado para verificar a influencia das alterações posturais na funcionalidade do apareIho respiratório de idosos, foi observada a diminuição da expansibilidade torácica na amostra ${ }^{28}$, corroborando com os resultados encontrados nesta pesquisa. Outra pesquisa que comparou a função respiratória entre adultos e idosos saudáveis demonstrou que há declínio da expansibilidade torácica com a idade avançada, sugerindo que o processo do envelhecimento do sistema respiratório na população estudada provocou grande impacto dessa variável analisada ${ }^{29}$. Neste estudo, a média da expansão da região abdominal foi a de menor valor obtido, que pode estar relacionada com as principais alterações encontradas na AP em bases pulmonares e a incidência pelo padrão respiratório intercostal superior. Sabe-se que o envelhecimento gera enfraquecimento dos músculos respiratórios, ocasionando perda parcial da expansibilidade tóraco-abdominal e possível modificação no padrão respiratório ${ }^{4,11,12}$

Quanto ao PFE e a PEmáx, foi possível estabelecer uma correlação linear, porém não significante. Não foram encontrados estudos que correlacionassem essas duas variáveis em idosos infectados pelo HIV, porém pesquisas realizadas com idosos demonstram que os valores absolutos de PFE, PEmáx e PImáx não atingem aqueles estimados ${ }^{29}$. Oskivig ${ }^{30}$ explica que com o passar dos anos ocorre o alargamento dos ductos alveolares e dos bronquíolos respiratórios, e, como consequência, o efeito negativo nas trocas gasosas e no fluxo expiratório forçado. Observa-se que o PFE tem uma diminuição média anual de 9,2L/min em idosos do sexo masculino e de $6 \mathrm{~L} / \mathrm{min}$ do gênero feminino ${ }^{31}$. Quanto à força muscular respiratória, há um estudo ${ }^{32}$ que avaliou homens e mulheres com idade entre 40 e 89 anos e verificou menores valores de PImáx e PEmáx nas mulheres em relação aos homens de mesma idade, e redução progressiva e significativa com o avançar de cada década nos valores das pressões respiratórias em ambos os gêneros. No presente estudo, os valores absolutos abaixo do previsto na literatura de PFE, PEmáx e PImáx foram de idosos homens ( $n=2)$.

As médias dos valores de dispneia, tanto no repouso quanto ao esforço, indicaram grau leve percebido pelos voluntários. Na literatura, há relatos de que, ao longo da epidemia da AIDS, a fadiga tem sido um dos sintomas mais comuns por pessoas infectadas $^{33}$. Dispneia e sensação de cansaço são sintomas que comumente estão relacionados com problemas cardiovasculares $^{12}$, que na população idosa podem configurar alterações severas de ordem sistêmica influenciadas pelo envelhecimento fisiológico do organismo. E o que percebe-se, conforme relatos da literatura, é que esses fatores, associados à infecção pelo HIV e à administração de medicamentos da TARV, podem implicar em comprometimentos cardíacos e pulmonares mais rapidamente manifestados nos idosos portadores da AIDS.

\section{Conclusão}

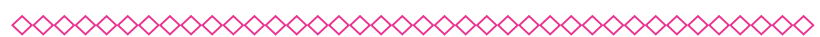

Os resultados deste estudo sugerem que idosos portadores da síndrome da imunodeficiência adquirida apresentam alterações cardiorrespiratórias. Mesmo com uma amostra reduzida, foi possível encontrar dados estatisticamente significativos do comportamento da PAS e FC e do PFE e PEmáx, que provavelmente podem estar relacionados ao processo de envelhecimento dos indivíduos e à administração de medicamentos para TARV.

Constata-se a importância deste estudo, uma vez que consegue abordar o idoso com HIV de forma multidimensional voltada para o sistema cardiorrespiratório. Sugerem-se pesquisas que avaliem a reabilitação cardíaca e respiratória no âmbito fisioterapêutico, para exaltar a importância da atuação deste profissional à população idosa portadora da AIDS. 
É importante colocar que devido a alguns participantes estarem preocupados com o sigilo de identidade e o próprio preconceito quanto à doença, alguns demonstram-se receosos a aceitarem a participação, sendo uma limitação do estudo. Contudo, após a explanação e o entendimento da pesquisa estes submeteram-se a avaliação.

\section{Referências}

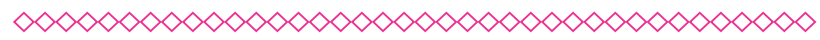

1. Carvalho, JAM; Garcia, RA. O envelhecimento da população brasileira: um enfoque demográfico. Cad. Saúde Pública. 2003;19(3):725-733.

2. Veras R. Fórum. Envelhecimento populacional e as informações de saúde do PNAD: demandas e desafios contemporâneos. Introdução. Cad. Saúde Pública. 2007;23(10):2463-2466.

3. Mazo, ZG; Lopes, AM; Benedetti, BT. Atividade fisica e o idoso: Concepções Gerontológicas. Porto Alegre: Sulina, ed 3a , 2009, p. 32-56.

4. Carvalho Filho, ET; Papaléo Neto, M. Geriatria: fundamentos, clínica e terapêutica. São Paulo: Atheneu, 2006.

5. Ávila WRM. Efeitos agudos do exercício resistido sobre linfócitos totais, CD4 e CD8 de idosas [Dissertação de Mestrado]. Brasília: Universidade Católica de Brasília (UCB); 2006.

6. Brasil. Ministério da Saúde. Secretaria de vigilância em saúde. Programa nacional de DST e AIDS. Plano Estratégico: programa nacional de DST e AIDS 2005. Brasília: Ministério da Saúde, 2005.

7. Neri, AL; (Org.) Idoso no Brasil: vivencias, desafios e expectativas na terceira idade. São Paulo: Fundação Perseu Abramo, Edições SESC, 2007.

8. Mota ,M. A infecção VHI nos idosos. VII HIV/ AIDS Virtual Congress - O VIH/SIDA na criança e no idoso. 2007.

9. Umphred, DA. Reabilitação neurológica. São Paulo: Manole, 2004.

10. Kumar, V; Abbas, AK; Fauto, N. Patologia: bases patológicas das doenças. Rio de Janeiro: Elsevier, 2005.
11. Pryor, JA; Webber ,BA. Fisioterapia para problemas respiratórios e cardíacos. Rio de Janeiro: Guanabara Koogan, 2002.

12. Deturk, WE; Cahalin, LP. Fisioterapia cardiorrespiratória: baseada em evidencias. Porto Alegre: Artmed, 2007.

13. Vassoler, CA; Sarnamento, GJV. Avaliação fisioterapêutica em UTI. In: Sarnamento GJV. Fisioterapia respiratória no paciente crítico: rotinas clínicas. São Paulo: Manole, 2007.

14. Ide, MR; Caromano, FA; Dip, MAVB; Guerino, MR. Exercícios respiratórios na expansibilidade torácica de idosos: exercícios aquáticos e solo. Fisioterapia em Movimento. 2007;20(2):33-40.

15. Velloso, M; Costa, CP; Ozeki, CM. Métodos de mensuração da dispnéia: uma revisão de literatura. ConSCIENTIAE SAÚDE. Rev Cient., UNINOVE, SP. 2002; 1:35-39.

16. Kovelis, D; Segretti, NO; Probst, VS; Lareau, SC; Brunetto, AF; Pitta, F. Validação do modified pulmonary functional status and dyspnea questionnaire e da escala do medical research council para o uso em pacientes com doença pulmonar obstrutiva crônica no Brasil. J Bras Pneumol. 2008;34(12):1008-1018.

17. Teymeny, AA; Rissato, CP; Matos, LB; Boaventura, CM; Lopes, CR; Carvalho, EM. Pico de fluxo expiratório em voluntários de 50 a 80 anos. Fisioter. Bras. 2008;9(5):399-406.

18. Costa, D; Forti, EMP; Barbalho-Moulim, MC; Rasera-Junior, I. Estudo dos volumes pulmonares e da mobilidade toracoabdominal de portadoras de obesidade mórbida, submetidas à cirurgia bariátrica, tratadas com duas diferentes técnicas de fisioterapia. Rev Bras Fisioter. 2009;13(4):294-300.

19. Neder, JA; Andreoni, S; Lerario, MC; Nery, $L E$. Reference values for lung function tests. II. Maximal respiratory pressure and voluntary ventilation. Braz J Med Biol. 1999;32(6)719-727.

20. Luther, VP; Wilkin, AM. HIV infection in older adults. Clin Geriatr Med. 2007;27:567-583.

21. Brasil. Ministério da Saúde. Secretaria de Vigilância em Saúde - Departamento de DST, Aids 
e Hepaites Virais. Boletim epidemiológica DST e AIDS. Brasília: Ministério da Saúde, 2010.

22. Raso, V; Casseb, JSR; Duarte, AJ; Greve, JMA. Uma breve revisão sobre exercício físico e HIV/ AIDS R. Bras. Ci e Mov. 2007; 15(4): 115-126.

23. Sociedade Brasileira de Cardiologia. V Diretrizes Brasileiras de Hipertensão Arterial. Arq. Bras. Cardiol. 2007;89(3):24-79.

24. Veronesi, R; Focaccia, R. Tratado de infectologia. São Paulo: Atheneu, 2002.

25. Gobel, FL; Norstro, LA; Nelson, RR; Jorgensen, CR; Wang, $Y$. The rate-pressure product as an index of myocardial oxygen consumption during exercises in patients with angina pectoris.

Circulation. 1978;57:549-556.

26. Kramer, AS; Lazzarotto, AR; Sprinz, E; Manfroi, WC. Metabolic abnormalities, antiretroviral therapy and cardiovascular disease in elderly patients with HIV. Arq Bras Cardiol. 2009;93(5):519-526.

27. Rodrigues, RL; Domingos, $\mathrm{H}$; Cunha, RV; Paniago, AM; Souza, AS. Risco cardiovascular pré e pós-terapia antirretroviral potente nos pacientes com síndrome da imunodeficiência adquirida. Rev Bras Clin Med. 2009; 7:153-160.
28. Pettenon, R; Milano, D; Bittencourt, DC; Schneider, RH. Adaptação funcional do aparelho respiratório e da postura no idoso. RBCEH, Passo Fundo. 2008;5(2):64-77.

29. Ruivo, S; Viana, P; Martins, C; Baeta, C. Efeito do envelhecimento cronológico na função pulmonar: Comparação da função respiratória entre adultos e idosos saudáveis. Rev Port Pneumol. 2009;15(4):629-653.

30. Oskvig, RM. Special problems in the Elderly. Chest. 1999;115(5 Suppl):158S-64S.

31. Cook, NR; Evans, DA; Scherr, PA; Speizer, FE; Vedal, S; Branch, LG, et al. Peak expiratory flow rate in an elderly population. Am J Epidemiol.1989;130(1):66-78.

32. Simões, RP; Auad, MA; Dionísio, J; Mazzonetto, $M$. Influência da idade e do sexo na força muscular respiratória. Fisioterapia e Pesquisa. 2007; 14(1):36-41.

33. Siegel K, Bradley CJ, Lekas H-M. Causal attribution for fatigue among late middle-age and older adults with HIV infection. J Pain Symptom Manage. 2004;28:211-224. 


\section{Anexos}

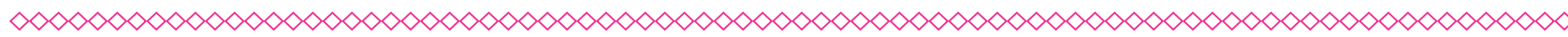

Tabela 1 - Valores absolutos, médias e desvios-padrão (DP) da cirtometria axilar, xifoideana e abdominal no repouso (Rep), inspiração máxima (Imáx) e Expiração máxima (Emáx)

\begin{tabular}{ccccccccccc} 
Idoso & \multicolumn{3}{c}{ Região axilar $(\mathrm{cm})$} & \multicolumn{3}{c}{ Região xifoideana $(\mathrm{cm})$} & \multicolumn{3}{c}{ Região abdominal (cm) } \\
\cline { 2 - 10 } 01 & Rep & Imáx & Emáx & Rep & Imáx & Emáx & Rep & Imáx & Emáx \\
02 & 91 & 93 & 90 & 90,5 & 91,5 & 90,5 & 88 & 90 & 87 \\
03 & 77,5 & 81,5 & 76 & 78 & 82 & 77 & 118 & 120 & 118 \\
04 & 116 & 120 & 116 & 118 & 120 & 118 & 109 & 109 & 108 \\
05 & 108 & 109 & 108 & 111 & 112 & 111 & 117 & 117 & 117 \\
06 & 95 & 96 & 93 & 96 & 96 & 96 & 91 & 91 & 91 \\
07 & 108 & 110 & 107 & 109 & 111 & 108 & 107,5 & 109,5 & 107 \\
08 & 97 & 99 & 96 & 96 & 98 & 96 & 95 & 95 & 95 \\
Média & 97,19 & 99,31 & 96,25 & 98,31 & 99,94 & 97,81 & 94,31 & 95,31 & 94 \\
DP & $\pm 12,91$ & $\pm 12,99$ & $\pm 13,38$ & $\pm 13,38$ & $\pm 13,11$ & $\pm 13,72$ & $\pm 16,31$ & $\pm 15,82$ & $\pm 16,19$ \\
\hline
\end{tabular}

Fonte: elaboração das autoras.

(clique para voltar ao texto)

Tabela 2 - Valores de médias e desvios-padrão (DP) dos maiores valores obtidos na avaliação do pico de fluxo expiratório (PFE), pressão expiratória máxima (PEmáx) e pressão inspiratória máxima (PImáx)

\begin{tabular}{ccccccc} 
Idoso & \multicolumn{2}{c}{ PFE (L/min)* } & \multicolumn{2}{c}{ PEmáx $(\mathbf{m m H g})^{*}$} & \multicolumn{2}{c}{ PImáx $(\mathbf{m m H g})$} \\
\cline { 2 - 6 } & $\begin{array}{c}\text { Valor } \\
\text { alcançado }\end{array}$ & $\begin{array}{c}\text { Valor } \\
\text { estimado* }\end{array}$ & $\begin{array}{c}\text { Valor } \\
\text { alcançado }\end{array}$ & $\begin{array}{c}\text { Valor } \\
\text { estimado }\end{array}$ & $\begin{array}{c}\text { Valor } \\
\text { alcançado }\end{array}$ & $\begin{array}{c}\text { Valor } \\
\text { estimado++ }\end{array}$ \\
1 & 590 & 430 & 80 & 78,39 & 60 & 80,51 \\
2 & 250 & 430 & 20 & 78,39 & 60 & 80,51 \\
3 & 340 & 417 & 60 & 75,95 & 100 & 78,55 \\
4 & 460 & 591 & 110 & 116,70 & 120 & 107,3 \\
5 & 190 & 515 & 20 & 102,93 & 10 & 93,7 \\
6 & 230 & 417 & 40 & 78,39 & 40 & 78,55 \\
7 & 260 & 591 & 120 & 115,89 & 120 & 106,5 \\
8 & 120 & 535 & 70 & 108,60 & 20 & 99,3 \\
Média & 305 & 490,75 & 65 & 93,75 & 66,25 & 90,12 \\
DP & $\pm 153,34$ & $\pm 76,44$ & $\pm 37,80$ & $\pm 17,00$ & $\pm 43,07$ & $\pm 11,85$
\end{tabular}

* De acordo com o manual do aparelho utilizado;

+ De acordo com Neder et al. (1999);

\# Correlação linear de Pearson entre as variáveis PFE e PEmáx $r=0,35(p=0,06)$

Fonte: elaboração das autoras.

(clique para voltar ao texto) 
Tabela 3 - Valores de médias e desvio-padrão (DP) do grau de dispneia no repouso e ao esforço

\begin{tabular}{ccc} 
Idoso & EAV & MRC \\
\hline 1 & 2 & 1 \\
2 & 2 & 1 \\
3 & 1 & 1 \\
4 & 0 & 0 \\
5 & 8 & 4 \\
6 & 2 & 1 \\
7 & 4 & 2 \\
8 & 5 & 3 \\
Média & 3 & 1,63 \\
DP & $\pm 2,56$ & $\pm 1,30$ \\
\hline
\end{tabular}

$E A V=$ Escala Analógica Visual;

MRC = Escala do Conselho Britânico de Pesquisas Médicas Modificada.

Fonte: elaboração das autoras.

(clique para voltar ao texto) 